\title{
High Resolution EFTEM and PEELS of Ga/GaAs Nano-"Ice Cream Cones" Grown by Dual-Wavelength Pulsed Laser Deposition
}

\author{
C.T. Schamp*, V.P. Oleshko*, W.A. Jesser*, B.S. Shivaram**, J.M. Howe* \\ *Department of Materials Science and Engineering, University of Virginia, 116 Engineer's Way, \\ Charlottesville, VA 22904 \\ **Department of Physics, University of Virginia, 382 McCormick Rd., Charlottesville, VA 22904
}

Assembled low-dimensional semiconductor nanostructures such as quantum dots, nanowires and nanotubes offer the possibility to realize various novel types of nanoscale electronic devices. In this work, we report on a new nanostructure denoted as nano-"ice cream cones" which is a $\mathrm{Ga} / \mathrm{GaAs}$ heterostructure fabricated using dual-wavelength pulsed laser deposition. High purity GaAs targets were ablated by dual wavelength laser pulses, $1064 \mathrm{~nm}$ and $532 \mathrm{~nm}$, from a frequency doubled, Qswitched Nd:YAG laser. By separating the wavelengths, the power of the fundamental $(1064 \mathrm{~nm})$ was found to be about $75 \%$ of the total. These wavelengths are co-linear in a single laser beam, resulting in the inherent alignment of the two wavelengths in contrast to the typical configuration of two separate lasers [1]. The laser beam was focused through a lens into a vacuum chamber and onto the GaAs target, ablating a plume of material normal to the target surface, which was collected on an amorphous carbon film supported by a $\mathrm{Cu}$ grid. Spectroscopic and structural characterization was performed using a JEOL 2010F analytical TEM equipped with a Schottky field-emission gun and operating at $197 \mathrm{kV}$ accelerating voltage with a point-to-point resolution of $0.23 \mathrm{~nm}$ and an information limit of $0.14 \mathrm{~nm}$. The instrument was equipped with a Gatan GIF 678 imaging filter and an Oxford ultrathin window EDXS detector.

The substrate temperatures investigated varied from room temperature to about $475^{\circ} \mathrm{C}$ over a range of laser energy densities incident on the target from about $25 \mathrm{~J} / \mathrm{cm}^{2}$ to $45 \mathrm{~J} / \mathrm{cm}^{2}$. Typical Ga/GaAs deposited particles were found to have a shape resembling an ice-cream cone 50-100 nm in length, as shown in Fig. a. This shape can be explained by the ejection of very hot liquid GaAs from the target. In flight, some of As preferentially evaporates from the molten ejected material, resulting in evaporative cooling [2]. Prior to impinging on the substrate, the material sufficiently cools allowing the GaAs to crystallize. The crystal facets and the particle takes on a morphology that minimizes interfacial and surface energies. The result is a liquid gallium segment of a sphere in contact with a faceted GaAs crystal. A low-loss PEEL spectrum of the liquid Ga sphere of $100 \mathrm{~nm}$ diameter in Fig. b shows a bulk plasmon at $13 \mathrm{eV}$, the $\mathrm{GaM}_{4,5}$-edge at $20 \mathrm{eV}$ and a double plasmon at $26 \mathrm{eV}$ energy loss, respectively. The corresponding PEEL spectrum of the solid GaAs cone reveals a surface plasmon at $10 \mathrm{eV}$, a broadened volume plasmon at $16 \mathrm{eV}$, followed by a shoulder at $21 \mathrm{eV}$ (the $\mathrm{GaM}_{4,5}$-edge) and a minor $\mathrm{AsM}_{4,5}$-edge at $41 \mathrm{eV}$ energy loss. Contrast tuning through energy filtering enabled separation of the $\mathrm{Ga}$ and $\mathrm{GaAs}$ phases at $20 \pm 1.5 \mathrm{eV}$ energy loss with enhanced intensity in the GaAs cone, and it indicated the presence of an As-containing shell of less than $10 \mathrm{~nm}$ thickness in Fig. a, which is confirmed through EDXS in Fig. c. A high-resolution EFTEM image at $16 \pm 1.5 \mathrm{eV}$ energy loss and an integrated line profile (inset 1) in Fig. d demonstrate an atomic scale view of the liquid Ga-solid GaAs interface along the [110] zone axis.

References:

[1] S. Witanachchi and P. Mukherjee, J. Vac. Sci. Technol. A 13 (1995) 1171.

[2] G.L. Hubbard, V.E. Denny and A.F. Mills, Int. J. Heat Mass Transfer. 18 (1975) 1003 
[3] This work was supported primarily through the MRSEC Center for Nanoscopic Materials Design by the National Science Foundation under Award Number DMR-0080016. VPO and JHM acknowledge support by DOE under Award DE-FG02-01ER4J918.

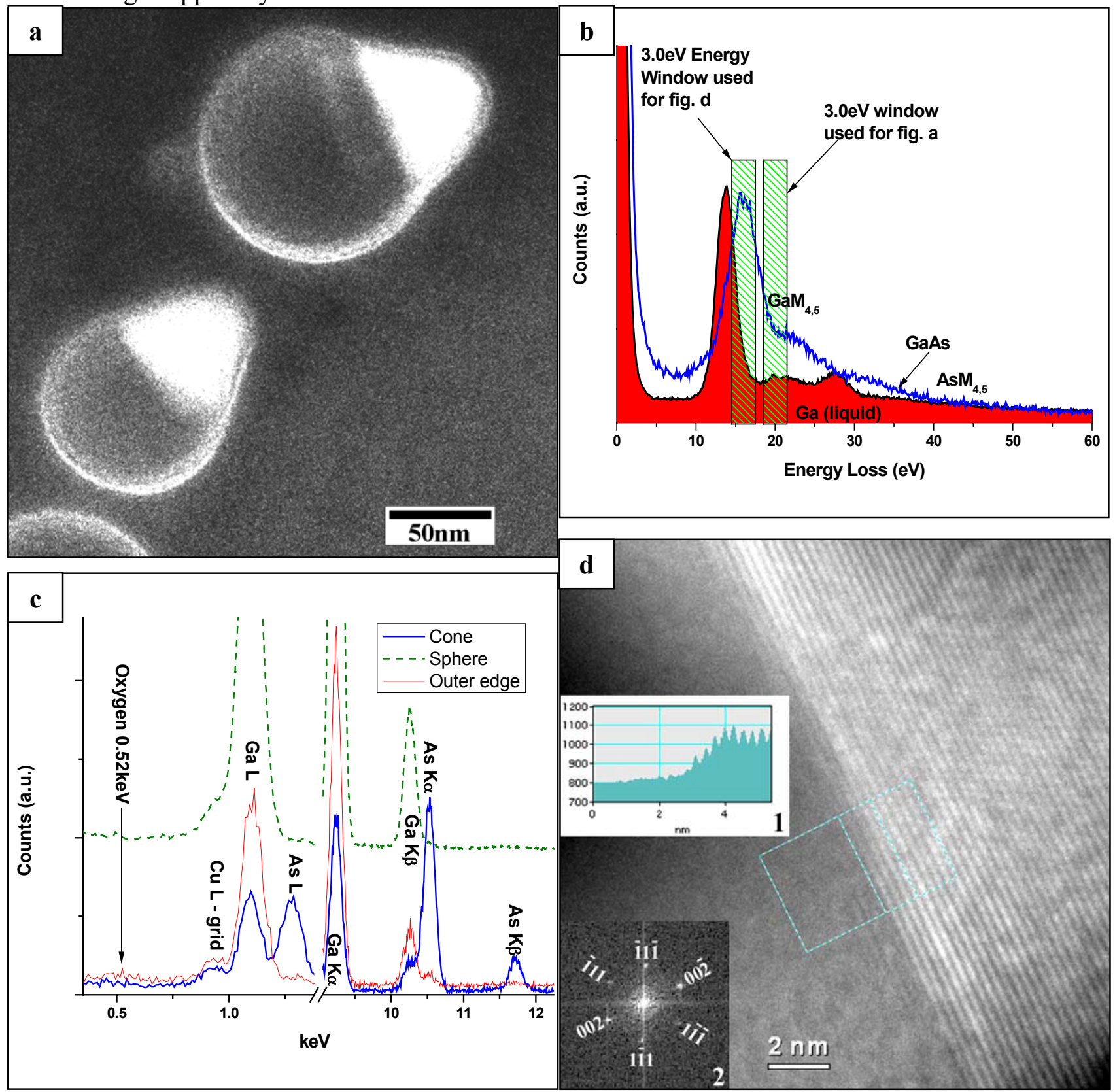

Fig. a. EFTEM image obtained using a $3.0 \mathrm{eV}$ slit centered at $20 \mathrm{eV}$ energy loss, showing solid/liquid "ice cream cone" nanoparticles.

Fig. b. Low-loss PEEL spectra from the center of the $100 \mathrm{~nm}$-sized liquid Ga sphere and the GaAs cone of larger particle near the top of Fig. a.

Fig. c. EDX spectra from an "ice cream cone" indicating a GaAs cone, a Ga sphere, and from the outer edge of the large particle shown in Fig. a, suggesting an As-containing shell.

Fig. d. High-resolution EFTEM image at $16 \pm 1.5 \mathrm{eV}$ energy loss showing the Ga-GaAs (liquid-solid) interface in the larger particle in Fig. a. Rectangular box indicates a line profile (inset 1) taken across the interface with a 100 pixel integration window. Inset 2 is an indexed digital diffractogram of the image from the cone, imaged along the $[110]$ zone axis. 